\title{
Clustering outdoor soundscapes using fuzzy ants
}

\author{
Bert De Coensel, Member, IEEE, Dick Botteldooren, Member, IEEE, \\ Kenny Debacq, Mats E. Nilsson and Birgitta Berglund
}

\begin{abstract}
A classification algorithm for environmental sound recordings or "soundscapes" is outlined. An ant clustering approach is proposed, in which the behavior of the ants is governed by fuzzy rules. These rules are optimized by a genetic algorithm specially designed in order to achieve the optimal set of homogeneous clusters. Soundscape similarity is expressed as fuzzy resemblance of the shape of the sound pressure level histogram, the frequency spectrum and the spectrum of temporal fluctuations. These represent the loudness, the spectral and the temporal content of the soundscapes. Compared to traditional clustering methods, the advantages of this approach are that no a priori information is needed, such as the desired number of clusters, and that a flexible set of soundscape measures can be used. The clustering algorithm was applied to a set of 1116 acoustic measurements in 16 urban parks of Stockholm. The resulting clusters were validated against visitor's perceptual measurements of soundscape quality.
\end{abstract}

\section{INTRODUCTION}

There is a growing awareness that outdoor areas with a high quality acoustic environment deserve special attention. For example, because rural and urban quiet areas have great potential for recreation and psychological restoration, their preservation and management have been addressed in the environmental noise directive of the European Community [1] and, consequently, also in policy documents of many countries. To support these policies, clear and objective procedures, well grounded in perception, are needed to assess environmental acoustic quality. However, it is known that multiple dimensions (loudness, spectrum, time, context, personal factors etc.) are involved in the perception of environmental sound [2], [3]. Soundscape research is a relatively new field of science which tries to tackle this problem in a holistic but human-centered way, concentrating on the way people consciously perceive their sonic environment [4]. A soundscape usually refers to the sound that can be heard in a natural, rural or urban outdoor environment, and it also encompasses the way it is perceived with consequential effects on quality of life, well-being and health.

It is commonly acknowledged that in order to find suitable soundscape quality indicators, it is necessary to combine information from physical, contextual and perceptual factors. A number of methods have been applied to relate physical indicators (derived from acoustic recordings/measurements) to perceptual-evaluative indicators of soundscape quality (derived from questionnaires or listening walks/measurements).

Bert De Coensel and Dick Botteldooren are with the Department of Information Technology (INTEC), Ghent University, Belgium (phone: +329-264-9994; fax: +32-9-264-9969; email: bert.decoensel@intec.ugent.be). Kenny Debacq is a former student at INTEC. Mats E. Nilsson and Birgitta Berglund are with the Gösta Ekman Laboratory for Sensory Research, Stockholm University and Karolinska Institute, Sweden.
Examples are multiple regression models, multidimensional scaling (distance or content models) and various clustering procedures.

In this paper, a novel algorithm for automatic classification of soundscape recordings is presented, based on several soft computing techniques. The proposed clustering approach (Section II) is inspired by the clustering behavior observed in colonies of several ant species. Compared to well known hierarchical or partitional clustering algorithms, such as $k$ means or the more general fuzzy $c$-means algorithms, ant clustering has the advantages that an initial estimate of the number of clusters does not have to be provided, and that a flexible set of soundscape measures can be used. The behavior of the ants, and as a consequence the number and composition of the resulting clusters, is governed by flexible fuzzy rules, which contain a rather large number of easily interpretable free parameters. This makes ant clustering very suitable for optimization, as compared to the more traditional clustering algorithms mentioned above. The fuzzy rules that govern the ant behavior are optimized using a specially adapted genetic algorithm in order to achieve an optimal set of homogeneous clusters. The similarity between two soundscapes is determined by the fuzzy resemblance of the shape of statistical distributions derived from the spectrogram. The proposed distance measure is purely based on physical measures, but obviously goes beyond the use of single-value measures such as the average (energy equivalent) A-weighted sound pressure level $\left(L_{\mathrm{Aeq}}\right)$ or the median sound level $\left(L_{\mathrm{A} 50}\right)$. The classification algorithm is applied to a large set of 10-minute soundscape recordings, made in 16 urban parks in Stockholm [5] (Section III). The results of the clustering of acoustic data are validated by visitor's questionnaire data on perceived soundscape quality [6]. Part of this validation has been reported earlier [7]; this paper will focus more thoroughly on the clustering algorithm itself.

\section{Methodology}

\section{A. Soundscape similarity}

An environmental sound recording can be physically characterized by its spectrogram, in which the frequency spectrum is plotted against time. However, directly comparing spectrograms is not very meaningful because of the stochastic nature of environmental sound, and moreover is not trivial when the recordings are of varying duration. A useful soundscape similarity measure will have to be based on statistical characteristics of the spectrogram. An analysis of several studies on soundscape perception [8] has shown that, in most cases, at least three principal components seem to arise in the way persons describe soundscapes: a factor related to 

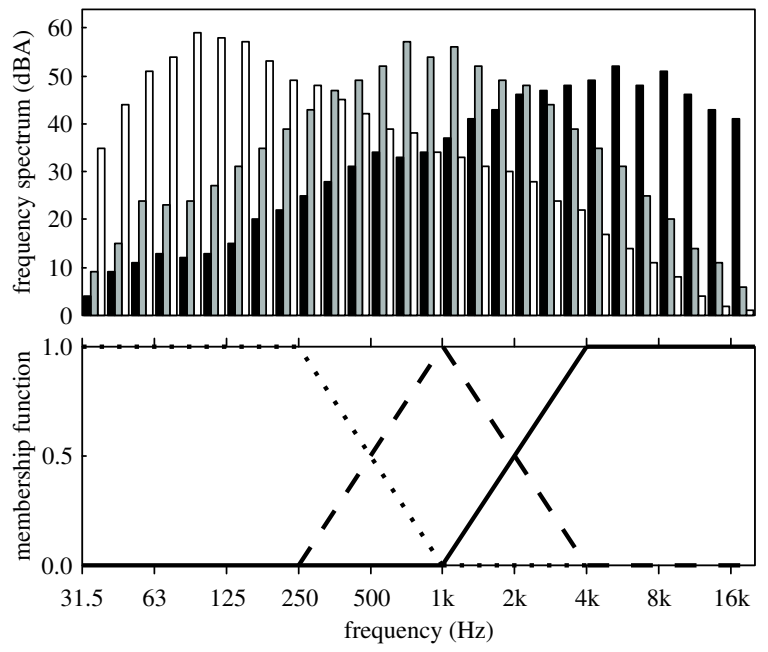

Fig. 1. Frequency spectra of three soundscape recordings (upper panel), together with an illustrative fuzzy partition of the frequency universe of discourse (lower panel). The sets shown could be labeled with the linguistic values "low" (dotted), "medium" (dashed) and "high" (solid) frequency.

loudness, a factor related to spectral content and a factor related to temporal structure. Therefore, three statistical distributions derived from the spectrogram of the soundscape were selected, representing the above mentioned factors: the A-weighted sound pressure level (SPL) histogram, the $1 / 3$-octave band frequency spectrum and the spectrum of temporal fluctuations. The last mentioned is calculated as the spectral density of the temporal envelope of time-varying loudness, and contains information on the rhythm of events in the sound (see [9]). Two soundscapes are defined to be similar when these three distributions are similar.

To quantify the similarity measure (distance measure), we note that each statistical distribution may be interpreted as a (discretized) fuzzy membership function [10], [11]. More in particular, the SPL histogram of a soundscape can be seen as a fuzzy set on the loudness universe of discourse, the frequency spectrum as a fuzzy set on the frequency universe of discourse, and the spectrum of temporal fluctuations as a fuzzy set on the universe of discourse of temporal content (rhythm). For the frequency spectrum, this is illustrated with an example in Figure 1. The three distributions have to be "vertically" rescaled to the interval $[0,1]$. Additionally, the SPL histogram is shifted "horizontally" to a common centre of gravity. This excludes the influence of the average SPL in the clustering algorithm, since it is desired that soundscapes with a similar histogram shape end up in the same cluster, rather than soundscapes with the same $L_{\text {Aeq }}$.

The notion of similarity is well-known in fuzzy set theory, and a large number of methods exist to quantify the resemblance of two fuzzy sets. Generally, a similarity measure is a binary fuzzy relation on the class of fuzzy sets on the universe of discourse considered (loudness, frequency and temporal content in our case) which yields a value in $[0,1]$. In [12], a distinction is made between measures inspired by set equality and measures inspired by the degree of compatibility or overlap. In [13], it is argued that it is often most worthwile to use an aggregation of several similarity measures. Therefore a hybrid similarity measure is proposed, which takes into account shape similarity and similarity of peak regions. More in particular, set equality is defined by [13]

$$
\begin{aligned}
\operatorname{Eql}\left(Z_{a}, Z_{b}\right)=T( & \inf _{x \in X} \mathcal{I}_{T}\left(Z_{a}(x), Z_{b}(x)\right), \\
& \left.\inf _{x \in X} \mathcal{I}_{T}\left(Z_{b}(x), Z_{a}(x)\right)\right)
\end{aligned}
$$

with $Z_{a}(x)$ and $Z_{b}(x)$ two fuzzy sets, $T$ a fuzzy $t$-norm and $\mathcal{I}_{T}$ a residual implicator, defined by

$$
\mathcal{I}_{T}(x, y)=\sup \{\gamma \in[0,1] \mid T(x, \gamma) \leq y\},
$$

whereas degree of compatibility is defined by

$$
\operatorname{Com}_{1}\left(Z_{a}, Z_{b}\right)=\frac{\sup _{x \in X} T\left(Z_{a}(x), Z_{b}(x)\right)}{\sup _{x \in X} S\left(Z_{a}(x), Z_{b}(x)\right)}
$$

or

$$
\operatorname{Com}_{2}\left(Z_{a}, Z_{b}\right)=\frac{\sum_{x \in X} T\left(Z_{a}(x), Z_{b}(x)\right)}{\sum_{x \in X} S\left(Z_{a}(x), Z_{b}(x)\right)}
$$

with $S$ a fuzzy $t$-conorm. Combining both approaches, the following hybrid measure for fuzzy set similarity is used (from [13]):

$$
\begin{aligned}
& \operatorname{Sim}\left(Z_{a}, Z_{b}\right)=\min ( \operatorname{Com}_{1}\left(Z_{a}, Z_{b}\right), \\
&\left.\max \left(\operatorname{Eql}\left(Z_{a}, Z_{b}\right), \operatorname{Com}_{2}\left(Z_{a}, Z_{b}\right)\right)\right)
\end{aligned}
$$

The similarity measure Sim is considered for the three fuzzy sets associated to each soundscape separately. The resulting three values are finally combined to obtain the global similarity measure for two soundscapes $a$ and $b$ :

$$
\begin{aligned}
E(a, b)=T & \left(\operatorname{Sim}\left(Z_{a}^{1}, Z_{b}^{1}\right),\right. \\
& \left.T\left(\operatorname{Sim}\left(Z_{a}^{2}, Z_{b}^{2}\right), \operatorname{Sim}\left(Z_{a}^{3}, Z_{b}^{3}\right)\right)\right)
\end{aligned}
$$

with $Z^{i}$ the fuzzy sets for loudness, spectral and temporal content. A combination of different $t$-norms and $t$-conorms was used (product, Zadeh or Łukasiewicz [11]) in Eqs. 1 to 4 , in order to achieve an optimal spread for the values of $E$ for a set of test soundscapes.

\section{B. Fuzzy ant clustering}

Ants are, because of their limited brain capacity, often assumed to reason only by means of rules of thumb [14]. However, the resulting behavior on the colony-level can be quite complex. A particular example is the clustering of the corpses of dead nestmates by taking only simple actions and without negotiating on where to gather the corpses. Because of the conceptual simplicity of this phenomenon, a computational clustering technique based on ant behavior was introduced by Deneubourg et al. [15], and was later refined by Monmarché [16], [17]. In this work, a slightly modified version of the algorithm by Schockaert et al. [18] is used, which is based on the work by Monmarché. In [18], 
fuzzy rules are used to control the behavior of the ants. Furthermore, no spatial relationship between clusters is considered (i.e. the clusters are kept in a list). The algorithm has been shown to work well on real-world datasets, giving comparable results as when using $k$-means or agglomerative hierarchical clustering, at a reduced computational load [18].

At the start of the algorithm, each soundscape is considered to form a cluster (heap) by itself. At each timestep of the algorithm, an artificial ant selects a heap at random, and undertakes one of the following actions. If it is carrying nothing, it can pick up a single item of the selected heap or it can pick up the entire heap. If it is carrying a load, it can drop the items it is carrying on the selected heap, thereby moving them to a different cluster, it can drop its load in an empty place, or it can decide to do nothing. The decision process is governed by a model of division of labour. A certain stimulus value $s$ and a response threshold value $t$ are associated with each task the ant may perform. The response threshold value is fixed, but the stimulus can change, and represents the need for an ant to perform the task. The probability that the ant starts performing the task is given by (from [18])

$$
p_{n}(s, t)=\frac{s^{n}}{s^{n}+t^{n}}
$$

where $n$ is a positive integer, $s \in[0,1]$ and $t \in] 0,1]$. For example, a loaded ant may drop its load, with a probability

$$
P_{\text {drop }}=p_{n_{i}}\left(s_{\text {drop }}, t_{\text {drop }}\right)
$$

where $i \in\{1,2\}$. When the ant is only carrying one item $n_{1}$ is used; otherwise $n_{2}$ is used. Similar probabilities are defined for the other possible cases.

For each cluster $H$, the center soundscape $c_{H}$ is defined as the soundscape which has the highest average similarity $E$ with all other soundscapes of the cluster. Note that this definition is independent of the problem at hand. The average similarity $A_{H}$ and the minimal similarity $M_{H}$ of a cluster $H$ are defined as

$$
A_{H}=\frac{1}{|H|} \sum_{v \in H} E\left(v, c_{H}\right)
$$

and

$$
M_{H}=\min _{v \in H} E\left(v, c_{H}\right)
$$

with $|H|$ the number of elements in $H$. When a load $L$ is considered, the average similarity between the center of $H$ and the items of $L$ can be estimated by

$$
B_{L, H}=T_{W}\left(E\left(c_{L}, c_{H}\right), A_{L}\right)
$$

with $T_{W}$ the Łukasiewicz $t$-norm [11] (for a formal proof, see [18]).

The values of the stimuli are calculated by evaluating a fuzzy rule base [18]. The stimulus for a loaded ant to drop its load $L$ on a heap $H$ is based on the average similarity $A_{H}$ and the estimation of the inter-cluster similarity $B_{L, H}$. If $B_{L, H}<A_{H}$, then the stimulus for dropping the load

\begin{tabular}{|c|c|c|c|c|c|}
\hline & \multicolumn{5}{|c|}{$\overline{A_{H}}$} \\
\hline & $\mathrm{VH}$ & $\overline{\mathrm{H}}$ & $M$ & $\mathrm{~L}$ & $\mathrm{VL}$ \\
\hline \multicolumn{6}{|c|}{$\begin{array}{l}\text { dropping the load } \\
B_{L, H}\end{array}$} \\
\hline $\mathrm{VH}$ & RH & $\mathrm{H}$ & $\mathrm{VH}$ & VVH & VVH \\
\hline $\mathrm{H}$ & L & RH & $\mathrm{H}$ & $\mathrm{VH}$ & VVH \\
\hline M & VVL & L & $\mathrm{RH}$ & $\mathrm{H}$ & $\mathrm{VH}$ \\
\hline $\mathrm{L}$ & VVL & VVL & $\mathrm{L}$ & $\mathrm{RH}$ & $\mathrm{H}$ \\
\hline $\mathrm{VL}$ & VVL & VVL & VVL & L & $\mathrm{RH}$ \\
\hline \multicolumn{6}{|c|}{$\begin{array}{l}\text { picking up a single item } \\
M_{H}\end{array}$} \\
\hline $\mathrm{VH}$ & $\mathrm{M}$ & - & - & - & - \\
\hline $\mathrm{H}$ & $\mathrm{H}$ & RH & - & - & - \\
\hline M & VVH & $\mathrm{VH}$ & $\mathrm{H}$ & - & - \\
\hline $\mathrm{L}$ & VVH & VVH & VVH & $\mathrm{VH}$ & - \\
\hline $\mathrm{VL}$ & VVH & VVH & VVH & VVH & $\mathrm{VVH}$ \\
\hline \multicolumn{6}{|c|}{$\begin{array}{l}\text { picking up an entire heap } \\
M_{H}\end{array}$} \\
\hline $\mathrm{VH}$ & $\mathrm{VVH}$ & - & - & - & - \\
\hline $\mathrm{H}$ & M & VH & - & - & - \\
\hline M & $\mathrm{L}$ & RL & $\mathrm{H}$ & - & - \\
\hline $\mathrm{L}$ & VVL & VL & $\mathrm{L}$ & $\mathrm{RH}$ & - \\
\hline VL & VVL & VVL & VVL & VL & $\mathrm{M}$ \\
\hline
\end{tabular}
should be low; if $B_{L, H} \geq A_{H}$, then the stimulus should be high. Because heaps should be able to grow, an ant
TABLE I

FUZZY RULeS TO INFER THE STIMULUS VALUES (From [18]).

should be allowed to drop its load when $B_{L, H} \approx A_{H}$. The rules are summarized in Table I. The linguistic terms used are very very high (VVH), very high $(\mathrm{VH})$, high $(\mathrm{H})$, rather high $(\mathrm{RH})$, medium $(\mathrm{M})$, rather low (RL), low (L), very low (VL) and very very low (VVL). An unloaded ant should pick up the most dissimilar item from a heap $H$, if the similarity between this item and $c_{H}$ is far less than the average similarity of the heap $A_{H}$. This means that by loading the item, the heap will become more homogeneous. An unloaded ant should only pick up an entire heap, if the heap is already homogeneous. Consequently, $A_{H}$ and $M_{H}$ are used to build similar rule bases for picking up single items and entire heaps (Table I). Although it can be tolerated that an ant wrongly picks up a single item from a homogeneous heap once in a while, it should be avoided that it picks up a heterogeneous heap. Therefore, the rules for picking up a single item are more strict than those for picking up an entire heap. For evaluating the fuzzy rules, the Sugeno fuzzy inference system [19] is used because of its calculation speed. The linguistic terms as shown in Table I are represented by triangular fuzzy sets.

The clustering algorithm only uses a single ant at each timestep. Because the ant uses only local information in his decision process and does not communicate, using multiple identical ants would have no advantages in a non-parallel implementation. However, parts of the clustering can be performed by ants with different parameters. More specifically, when $q$ different ants are used, each of them will cluster for $I / q$ iterations, with $I$ the total number of iterations. Note that, although sometimes wrongly assumed, ant clustering is not a consequence of swarm intelligence [20]; it is at most a form of self-organizing behavior [21]. 
The algorithm contains no stop criterion, so a predefined number of time steps $I$ are simulated, depending on the number of soundscapes to be clustered. Since the above sketched algorithm is probabilistic in nature, the clustering result will often contain "impurities", such as singleton clusters or clusters that are non-homogeneous due to a single item. These impurities are the result of the random nature of the ant behavior, and will always be present, regardless of the number of time steps used. Therefore, several post-clustering steps were introduced that "smooth out" the impurities, without having a global impact on the cluster composition.

In a first post-clustering step, all clusters that contain fewer elements than a given threshold number are removed and their elements are relocated to the best suiting remaining cluster. In a second post-clustering step, the least fitting item in each cluster is relocated (if necessary) to the best fitting cluster. In a third optimization step, the clustering results obtained in different clustering runs (10 in this algorithm) are merged. The merged clusters contain items which were clustered together in more than half of the separate clustering runs. The optimization steps were found to significantly enhance the homogeneity of the results.

\section{Ant behavior optimization using a genetic algorithm}

The ant clustering algorithm contains a number of parameters that influence the evaluation of the fuzzy rule bases and the behavior of the ant(s), and as a consequence the number and composition of the resulting clusters. Examples are the parameters $n_{i}$ in the evaluation of the chance for dropping the load, the various threshold values $t$ associated with each action, and the number of ants used. The performance of the clustering algorithm will therefore depend on the particular parameter values (11 in total for each ant). This makes the ant clustering algorithm very suitable for optimization.

To find the ant parameters resulting in an optimal clustering solution, a standard genetic algorithm (GA) [22] was used, slightly adapted to the peculiarities of the problem at hand. The genome of a clustering solution simply consists of the set of parameters of the ant(s) used, coded as floating point numbers. Since a variable number of ants may be used, the genome has a variable length, and consists of separate segments, each corresponding to the parameters of a single ant (the total number of clustering iterations $I$ is kept constant in order not to favor the use of a large number of different ants). The fitness of a clustering solution is evaluated using a fuzzy rule base. Let $N$ be the number of soundscapes in the dataset, and $|\Omega|$ the number of clusters in the clustering solution $\Omega$. An optimal clustering will have the following qualities:

- the average similarity within clusters, as defined by

$$
\frac{1}{|\Omega|} \sum_{H \in \Omega} A_{H}
$$

is high, where the linguistic term "high" is represented by the trapezoidal fuzzy set $(0.6,0.8,1,1)$;
- the minimal similarity within clusters, as defined by

$$
\min _{H \in \Omega} M_{H}
$$

is high, where the linguistic term "high" is represented by the trapezoidal fuzzy set $(0.4,0.6,1,1)$;

- the average similarity between clusters, as defined by

$$
\frac{1}{|\Omega|^{2}} \sum_{H_{1}, H_{2} \in \Omega} B_{H_{1}, H_{2}}
$$

is low, where the linguistic term "low" is represented by the trapezoidal fuzzy set $(0,0,0.5,0.7)$;

- the average size of the clusters, as defined by

$$
\frac{1}{|\Omega|} \sum_{H \in \Omega}|H|
$$

is within reasonable bounds, relative to the number of soundscapes, where the linguistic term "within reasonable bounds" is represented by the trapezoidal fuzzy set (2, $N / 10, N / 5, N / 2)$;

- the number of clusters $|\Omega|$ is within reasonable bounds, relative to the number of soundscapes, where the linguistic term "within reasonable bounds" is represented by the trapezoidal fuzzy set $(2,6, N / 10, N / 5)$.

Note that the fitness function is not deterministic - the same set of ants may produce clustering of different quality when used again - due to the stochastic nature of the ant clustering algorithm.

At the start of the genetic algorithm, a generation of random sets of ants is created, with a maximum of 5 ants for each set, and the corresponding clustering solutions are calculated. At each iteration, a new generation of offspring solutions is produced, which tries to combine the positive elements of the previous generation. Parents are selected for reproduction based on their fitness. A tournament selection procedure was used: a subgroup of $k$ clustering solutions is selected at random, and the best solution of this subgroup is elected to be a parent. The tournament size $k$ determines the selection pressure; a value of 2 was used. Offspring is then produced from parents by applying crossover ( 2 parents needed) or mutation (1 parent needed) operators. Each operator has the same probability to be used. Crossover operations implemented include exchanging single ant parameters or exchanging whole ants. Mutation operations implemented include changing a single parameter randomly and inserting a random ant or removing an ant randomly, if the solution uses multiple ants. An elitism survival mechanism is used: the next generation consists for $2 / 3$ of offspring, and for $1 / 3$ of the best solutions of the previous generation (the elitists).

The algorithm is terminated when the variance of the fitness within the current generation has become small, indicating that the algorithm has converged, when the best solution's fitness has reached a plateau such that successive iterations no longer produce better results, or when a maximum number of iterations is reached. 


\section{VALIDATION}

The classification algorithm was validated on a database of acoustic measurements, made in 16 urban parks in Stockholm [5]. These measurements were conducted in parallel with a questionnaire study. In total 1116 visitors answered questions on perceived soundscape quality and road traffic annoyance. While the questionnaires were conducted, the 1/3-octave band spectrogram was recorded at about the same location, resulting in a database of 1116 sound spectrogram registrations with associated quality assessments. The SPL histogram, frequency spectrum and the spectrum of temporal fluctuations were calculated for each 10-minute soundscape, based on the recorded spectrograms, and formed the input for our clustering algorithm.

The ant parameters for clustering were optimized using the described genetic algorithm. A single generation consisted of 100 solutions, and the genetic algorithm was run for 65 generations. Figure 2 shows the average and maximum clustering fitness within each generation. From the graph it could be concluded that the algorithm did not converge: generation 65 still consisted of a broad range of good and less good clustering results, reflected in the difference between maximum and average fitness. The maximum fitness reached a plateau at about the 40th generation. However, most solutions in the 65th generation had about the same genome; small variations resulted in a drop in fitness, explaining the lower average. The best solution encountered (in generation 56) made use of three ants, two of which were very similar (only one parameter differing slightly), and was used to produce the clustering results described in the next section. A total of 49 clusters were obtained, with an average of 23 soundscapes in each cluster.

Figure 3 shows graphs relating various perceptual soundscape indicators, averaged over all soundscapes within each cluster. Each dot represents one of 49 clusters of soundscapes. From the trends, some expected conclusions can be drawn, e.g. soundscape quality in urban parks is strongly related to the presence of natural sound $\left(r^{2}=0.36\right)$ and the absence of mechanical sound $\left(r^{2}=0.66\right)$. Figure 4 shows the same graphs, but without using the proposed clustering algorithm, i.e. soundscapes are randomly put together into

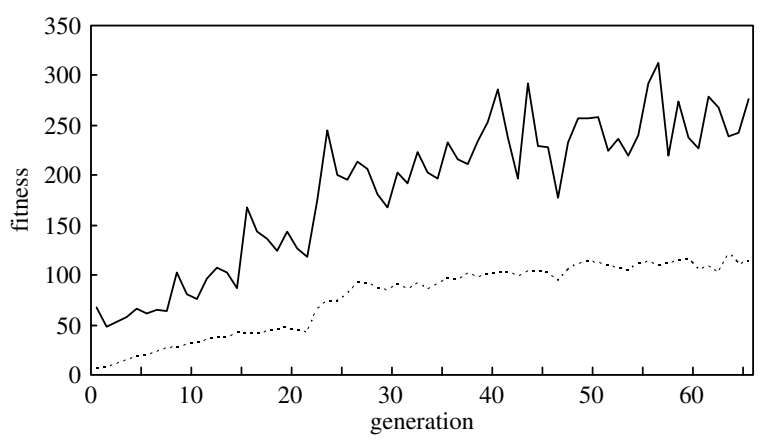

Fig. 2. Maximum (solid line) and average (dashed line) fitness in the GA.
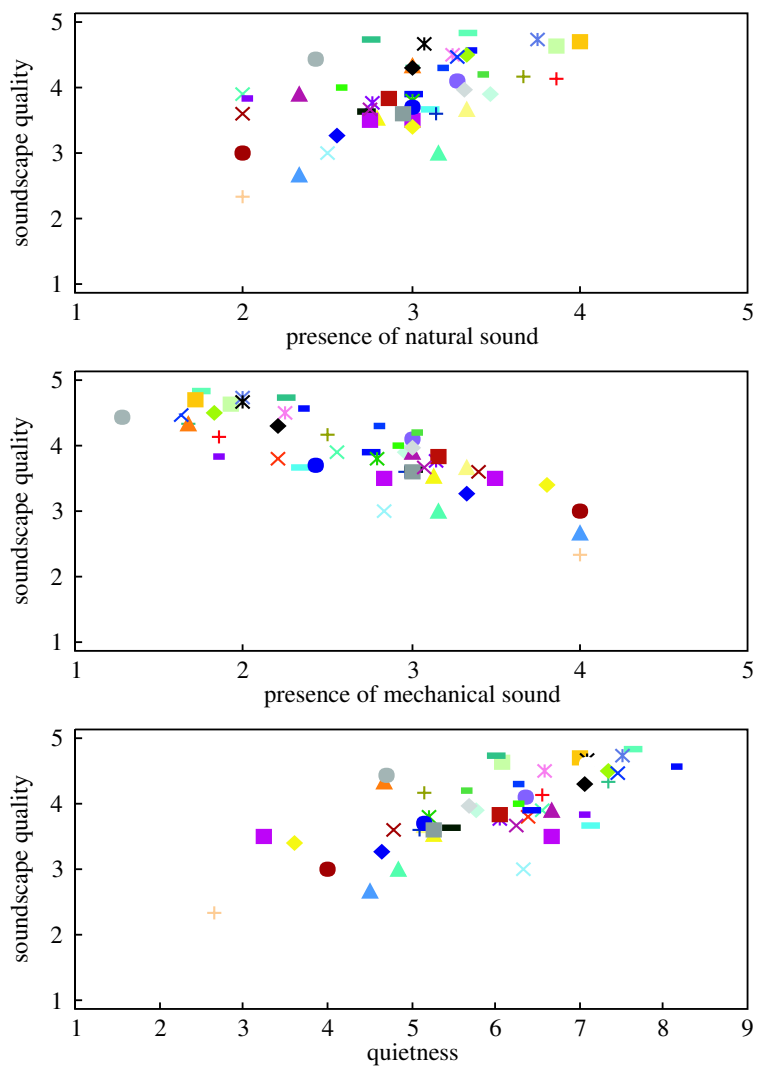

Fig. 3. Scatter plots of various perceptual attribute scales of soundscapes, averaged within clusters (shown as dots) obtained using the ant algorithm. Soundscape quality, presence of natural sound and presence of mechanical sound were assessed on a 5-point scale, quietness on a 9-point scale.

49 clusters. From the broader variance in the position of the dots in Figure 3, corresponding to various clusters, it can be concluded that by clustering based on statistical physical parameters only, (part of) the variation between soundscapes observed in questionnaire results can be reproduced. In other words, our algorithm is able to make a differentiation among soundscapes, which simulates the visitor's soundscape differentiations as measured with the perceptual attribute scales of the questionnaire.

A stepwise multiple linear regression analysis was performed, with perceived soundscape quality (assessed on a 5-point scale) as dependent variable, and with the presence of (i) sound caused by other people, (ii) natural sound and (iii) mechanical sound (assessed on a 5-point scale), and the perceived (iv) pleasantness, (v) eventfulness, (vi) excitingness and (vii) quietness of the soundscape (assessed on a 9-point scale) as independent variables. Table II summarizes the results of the final model, which explains about $76 \%$ of the variance in perceived soundscape quality. It is found that a high quality soundscape is characterized by quietness, the presence of natural sound, the absence of a lot of sound events and the absence of mechanical sounds - a result that 

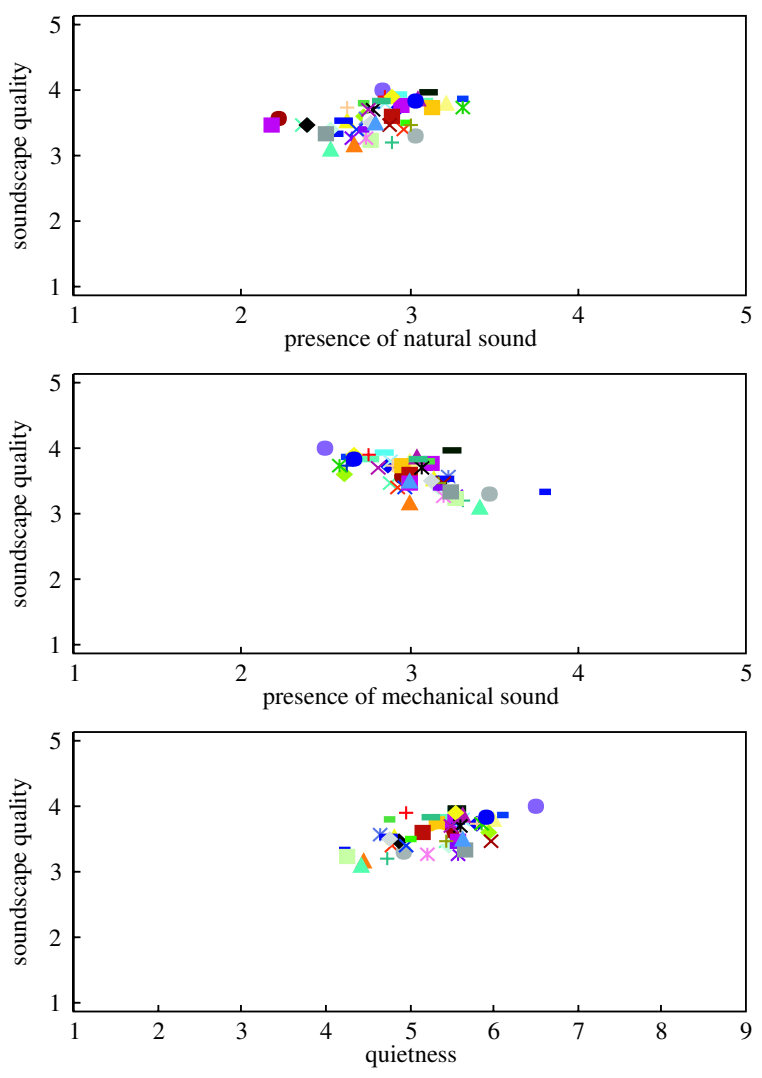

Fig. 4. Same plots as in Figure 3, but randomly clustered instead of using the fuzzy ant algorithm.

TABLE II

STEPWISE MULTIPLE LINEAR REGRESSION ANALYSIS OF PERCEPTUAL SOUNDSCAPE ATTRIBUTES ON PERCEIVED SOUNDSCAPE QUALITY.

\begin{tabular}{lc}
\hline \hline Independent variable & Coefficient \\
\hline constant & $3.992^{* * *}$ \\
Presence of mechanical sound & $-0.403^{* * *}$ \\
Presence of natural sound & $0.330^{* *}$ \\
Quietness & $0.122^{*}$ \\
Eventfulness & $-0.119^{*}$ \\
\hline \hline${ }^{*} p<0.05,{ }^{* *} p<0.01,{ }^{* * *} p<0.001$.
\end{tabular}

could be expected for an urban park context.

The proposed ant clustering algorithm has the main advantage that clusters aggregate soundscapes with similar statistical loudness, spectral and temporal properties. Outlier clusters can be used to trace for specific physical properties of the soundscape that may have modified perception. Figure 5 shows the psychophysical relationship between road traffic noise annoyance and $L_{\mathrm{Aeq}}$ for the 49 soundscape clusters. The graph shows the expected trend in exposure and effect; however, there is a large spread. Figure 6 shows the fuzzy membership functions based on the A-weighted SPL histogram for several outlier clusters, marked A to

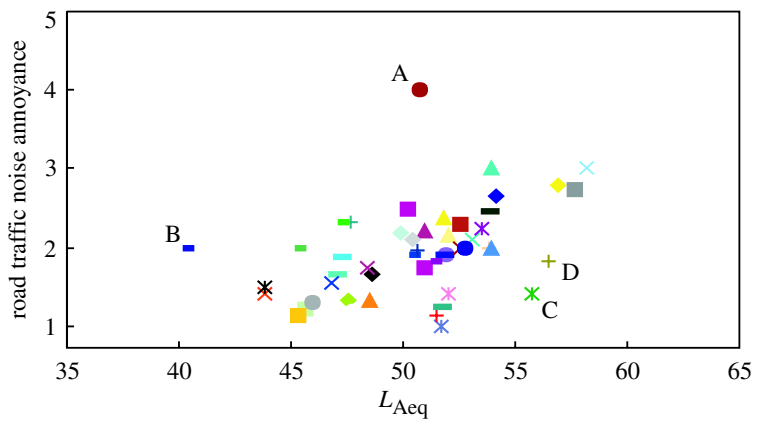

Fig. 5. Scatter plot of perceived road traffic noise annoyance (assessed on a 5-point scale) as a function of $L_{\mathrm{Aeq}}$, averaged within clusters.
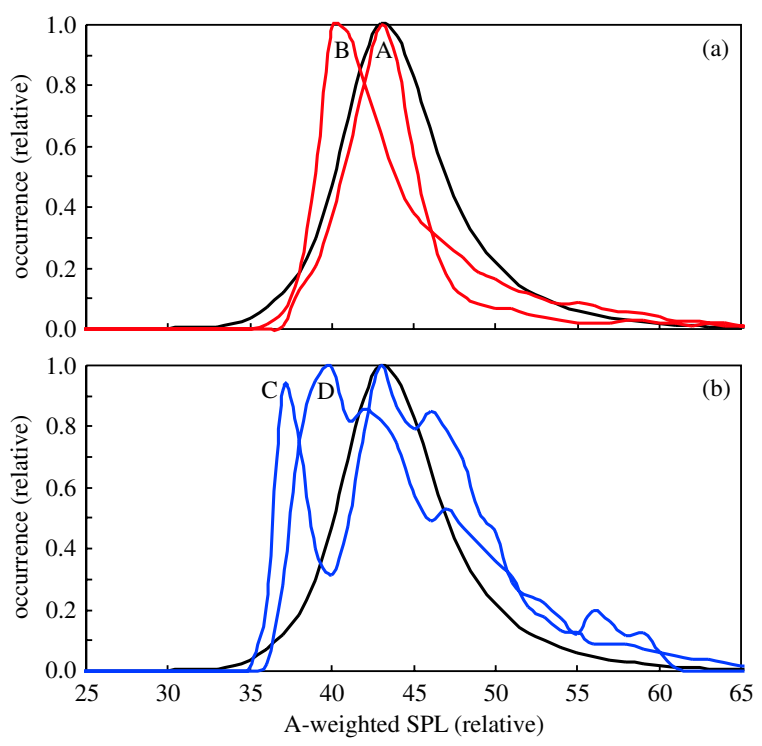

Fig. 6. Fuzzy membership function based on the mean A-weighted SPL histogram of several outlier clusters (marked in Figure 5) compared to the mean histogram of the remaining clusters (in black).

$\mathrm{D}$ in Figure 5, together with the average histogram of the remaining clusters. Note that the histograms were shifted horizontally to obtain a common centre of gravity, thus the SPL levels are relative. Clusters A and B aggregate soundscapes which were found to be more annoying due to road traffic noise than can be expected on the basis of the $L_{\text {Aeq }}$; clusters $\mathrm{C}$ and $\mathrm{D}$ aggregate soundscapes which were found to be less annoying. Compared to the mean, the histogram of clusters A and B is narrower. This indicates a "grey" or low fidelity soundscape [23], with few dynamics, foreground and background. Compared to the mean, the histograms of clusters $\mathrm{C}$ and $\mathrm{D}$ are much wider, and show a peak at lower levels. This indicates a (relatively) low background noise level with noise events which can have a high level (high fidelity soundscape). If anything, the analysis shows that neither $L_{\mathrm{Aeq}}$ nor $L_{\mathrm{A} 50}$ are sufficient predictors of noise annoyance. 


\section{CONCLUSiONS}

In this paper a novel approach was outlined for clustering environmental sound recordings or "soundscapes". Soundscape similarity is based on fuzzy resemblance of the SPL histogram, the frequency spectrum and the spectrum of temporal fluctuations. The algorithm is based on ant clustering, in which the ants obey fuzzy rules optimized using a genetic algorithm, in order to obtain a well balanced clustering result. Compared to traditional clustering algorithms, this approach has the added advantage of not needing any a priori information. The algorithm was tested on a large set of soundscape recordings in urban parks in Stockholm. It was shown that clustering purely on physical parameters can mimic part of the true variation found in perceptual characterizations of soundscapes. Furthermore, it was shown how outliers can be used to trace for specific physical properties of soundscapes that may modify soundscape perception.

\section{ACKNOWLEDGMENT}

The field study of soundscape recordings was conducted in the Swedish research programme "Soundscape Support to Health" supported from the Foundation for Strategic Research (MISTRA), the Road Administration and the Agency for Innovation Systems (Vinnova).

\section{REFERENCES}

[1] Directive 2002/49/EC of the European Parliament and of the Council relating to the assessment and management of environmental noise, Jun. 2002.

[2] S. Viollon and C. Lavandier, "Multidimensional assessment of the acoustic quality of urban environments," in Proceedings of The 29th International Congress on Noise Control Engineering (Inter-noise), Nice, France, Aug. 2000

[3] J. D. Guillén and I. López Barrio, "Importance of personal, attitudinal and contextual variables in the assessment of pleasantness of the urban sound environment," in Proceedings of The 19th International Congress on Acoustics (ICA), Madrid, Spain, Sep. 2007.

[4] Acta Acustica united with Acustica, vol. 92, no. 6, 2006, special issue on soundscapes.

[5] M. E. Nilsson, D. Botteldooren, and B. De Coensel, "Acoustic indicators of soundscape quality and noise annoyance in outdoor urban areas," in Proceedings of The 19th International Congress on Acoustics (ICA), Madrid, Spain, Sep. 2007.

[6] M. E. Nilsson and B. Berglund, "Soundscape quality in suburban green areas and city parks," Acta Acustica united with Acustica, vol. 92, no. 6, pp. 903-911, 2006.
[7] B. De Coensel, D. Botteldooren, K. Debacq, M. E. Nilsson, and B. Berglund, "Soundscape classifying ants," in Proceedings of The 2007 Congress and Exposition on Noise Control Engineering (Inter-noise), Istanbul, Turkey, Aug. 2007.

[8] B. De Coensel and D. Botteldooren, "The quiet rural soundscape and how to characterize it," Acta Acustica united with Acustica, vol. 92, no. 6, pp. $887-897,2006$

[9] D. Botteldooren, B. De Coensel, and T. De Muer, "The temporal structure of urban soundscapes," J. Sound. Vib., vol. 292, no. 1-2, pp. $105-123,2006$

[10] L. A. Zadeh, "Fuzzy sets," Information and Control, vol. 8, no. 3, pp. 338-353, 1965.

[11] D. Dubois and H. Prade, Eds., Fundamentals of Fuzzy Sets. Springer, 2000.

[12] E. Tsiporkova and H.-J. Zimmermann, "Aggregation of compatibility and equality: A new class of similarity measures for fuzzy sets," in Proceedings of the 7th International Conference on Information Processing and Management of Uncertainty in Knowledge-based Systems (IPMU), vol. II, Paris, France, 1998, pp. 1769-1776.

[13] C. Cornelis, M. De Cock, D. Botteldooren, and E. E. Kerre, "Fuzzy set theory: a useful interlingua?" in Proceedings of the 5th International Conference on Knowledge-based Intelligent Information Engineering Systems and Allied Technologies (KES), Osaka, Japan, Sep. 2001, pp. 1137-1141.

[14] B. Hölldobler and E. O. Wilson, The ants. Heidelberg, Germany: Springer-Verlag, 1990.

[15] J. L. Deneubourg, S. Goss, N. Franks, A. Sendova-Franks, C. Detrain, and L. Chrétien, "The dynamics of collective sorting robot-like ants and ant-like robots," in Proceedings of the 1st International Conference on Simulation of Adaptive Behavior on From Animals to Animats, Paris, France, 1990, pp. 356-363.

[16] N. Monmarché, M. Slimane, and G. Venturini, "On improving clustering in numerical databases with artificial ants," in Proceedings of the 5th European Conference on Artificial Life (ECAL'99), Swiss Federal Institute of Technology, Lausanne, Switzerland, Sep. 1999.

[17] N. Monmarché, "Artificial ant based algorithms applied to clustering and optimization problems (in French)," Ph.D. dissertation, François Rabelais University, Tours, France, Dec. 2000.

[18] S. Schockaert, M. De Cock, C. Cornelis, and E. E. Kerre, "Clustering web search results using fuzzy ants," International Journal of Intelligent Systems, vol. 22, no. 5, pp. 455-474, 2007.

[19] M. Sugeno and G. T. Kang, "Structure identification of fuzzy model," Fuzzy Sets and Systems, vol. 28, no. 1, pp. 15-33, 1988.

[20] E. Bonabeau, M. Dorigo, and G. Theraulaz, Swarm Intelligence: From Natural to Artificial Systems, ser. Santa Fe Institute Studies in the Sciences of Complexity. New York, USA: Oxford University Press, 1999.

[21] S. Camazine, J.-L. Deneubourg, N. R. Franks, J. Sneyd, G. Theraulaz, and E. Bonabeau, Self-organization in Biological Systems. Princeton, New Jersey, USA: Princeton University Press, 2003.

[22] M. Mitchell, An Introduction to Genetic Algorithms. Cambridge, Massachusetts, USA: The MIT Press, 1998.

[23] R. M. Schafer, The soundscape: Our Sonic Environment and the Tuning of the World. Rochester, Vermont, USA: Destiny Books, 1994. 


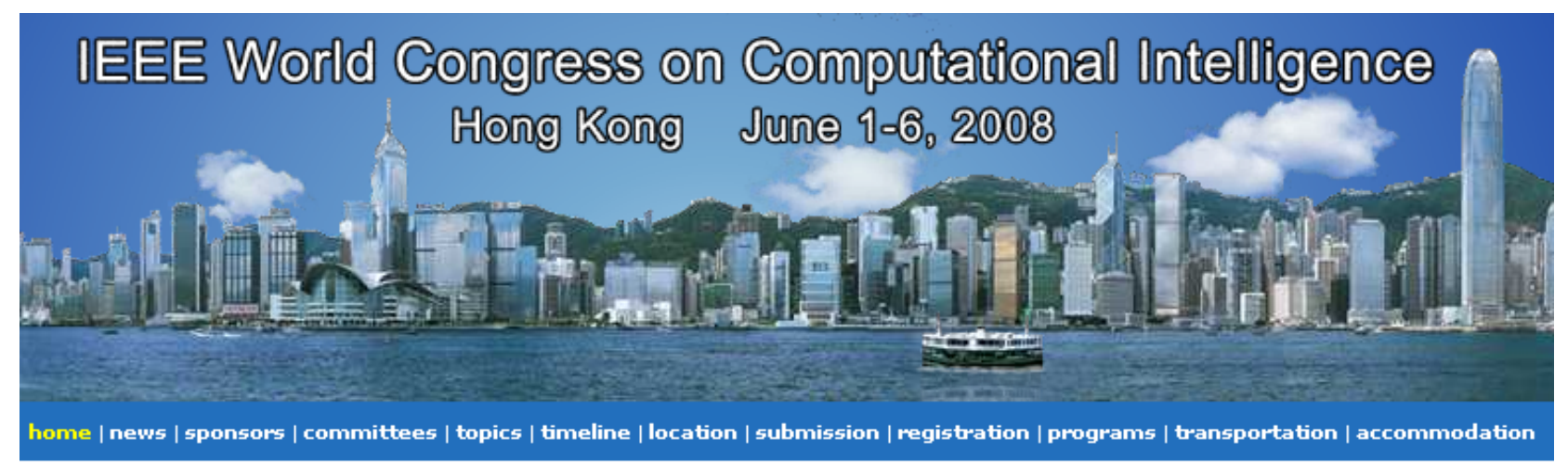

Messages from General Chair

On behalf of the Organizing Committee, I would like to thank you to participate in the 2008 IEEE World Congress on Computational Intelligence $(\mathrm{WCCl} 2008$ ). WCCl 2008 marks the fifth milestone in this series with a glorious history from $\mathrm{WCCl} 1994$ in Orlando, WCCl 1998 in Anchorage, $\mathrm{WCCl} 2002$ in Honolulu, to $\mathrm{WCCl} 2006$ in Vancouver. Sponsored by the IEEE Computational Intelligence Society and cosponsored by the International Neural Network Society, Evolutionary Programming Society, and the Institution of Engineering and Technology, $\mathrm{MCCl} 2008$ is the joint event of 2008 International Joint Conference on Neural Networks (IJCNN 2008), 2008 IEEE International Conference on Fuzy Systems (FUZZ-IEEE 2008), and 2008 IEEE Congress on Evolutionary Computation (CEC 2008). WCCl 2008 features the state-of-the-art plenary and invited speeches, parallel regular sessions, focused special sessions, interactive poster sessions, moderated panel discussions, informative pre-congress tutorials, complementary post-congress workshops, and entertaining social functions with following highlights:

- Five plenary speeches by world authorities and award recipients.

- Fifteen invited talks by renowned experts.

- Nine pre-congress tutorials by leading researchers in the fields with much reduced registration fees for widespread educational purposes.

- 2518 submissions from authors of over 70 countries across all inhabitable continents.

- Seventy nine advanced special sessions organized by domain specialists on focused topics.

- Eleven panels on a variety of topics to allow in-depth discussions and close interactions.

- Ten competitions in four categories on games, navigation, data mining, and optimization.

- Three post-congress workshops in Hong Kong and Macao for additional opportunities.

WCCl 2008 aims to provide a stimulating forum for thousands of scientists, engineers, educators, and students from all over the world to disseminate the latest research findings and exchange information on emerging areas of research in the fields. WCCl 2008 also creates a pleasant environment for you to meet your old friends for refreshing your memories and make new friends for expanding your network connections with possible future collaborations. Social events include a welcome reception, a women-in-computational-intelligence reception, an award banquet, a student and young graduate reception, and a farewell party. During the six days, we achieved a lot intellectually via intensive interactions and information exchanges. 